\title{
Preservation of sperm of cancer patients: extent of use and pregnancy outcome in a tertiary infertility center
}

\author{
Amnon Botchan, Shiri Karpol, Ofer Lehavi, Gedalia Paz, Sandra E Kleiman, Leah Yogev, Haim Yavetz \\ and Ron Hauser
}

Sperm cryopreservation is the best modality to ensure future fertility for males diagnosed with cancer. The extent to which cryopreserved sperm is actually used for impregnation, the fertility treatment options that are available and the success rates of these treatments have not been investigated in depth. The medical records of 682 patients who cryopreserved sperm cells due to cancer treatment were analyzed. Seventy of these patients withdrew their frozen sperm for fertility treatments over a 20-year period (most within the first 4 years after cryopreservation). Sperm quality of different malignancies and outcomes of assisted reproduction treatment (ART) for pregnancy achievement in relation to the type of treatment and the type of malignancy were evaluated. The results showed that the rate of using cryo-thawed sperm from cancer patients for fertility treatments in our unit was $10.3 \%$. Sperm quality indices differed between different types of malignancies, with the poorest quality measured in testicular cancer. Conception was achieved in 46 of the 184 ART cycles (25\%), and resulted in 36 deliveries. The use of intracytoplasmic sperm injection (ICSI) methodology yielded a significantly higher pregnancy rate (37.4\%) than intrauterine insemination (IUI; $11.5 \%$ ) and was similar to other groups of infertile couples using these modalities. In vitro fertilization (IVF) failed to produce pregnancies. In conclusion, the rate of use of cryopresseved sperm in cancer patients is relatively low (10.3\%). Achievement of pregnancies by ICSI presents the best option but when there are enough stored sperm samples and adequate quality, IUI can be employed. Cryopreservation is nevertheless the best option to preserve future fertility potential and hope for cancer patients.

Asian Journal of Andrology (2013) 15, 382-386; doi:10.1038/aja.2013.3; published online 25 March 2013

Keywords: assisted reproduction treatment (ART); cancer; pregnancy outcome; sperm cryopreservation

\section{INTRODUCTION}

Life expectancy of cancer patients has markedly improved, but many problems associated with their quality of life have not been solved and some have even grown worse. One of the major concerns of young male cancer patients is the hazard to their fertility potential caused by the oncological treatment. ${ }^{1}$ It has been known for years that various oncological treatment modalities might endanger sperm production. ${ }^{2-9}$

The measure of damage as well as the recovery potential is governed by numerous factors. These include the sperm quality before treatment, the type of malignancy and the therapeutic regimen used, e.g., type, dosage and duration of the treatment. ${ }^{9,10}$ There is no single criterion for predicting the recovery of sperm production. ${ }^{11}$

Currently, however, the only way to preserve reproductive potential in male cancer patients remains sperm cryopreservation, followed by the implementation of various assisted reproduction treatments (ARTs) for conception.

In most cases, sperm cryostorage takes place before initiating cancer treatment. Because a relatively small number of patients make use of their frozen sperm, there are very few published evidence-based data on the ART outcome of these sperm for pregnancy achievement, thus calling into doubt the benefit of routine sperm cryostorage for young cancer patients. Furthermore, there are different approaches to the best use of these sperm. Some choose a specific ART to be used (i.e., intrauterine insemination (IUI), in vitro fertilization (IVF) or intracytoplasmic sperm injection (ICSI)) according to sperm quantity and quality, ${ }^{12,13}$ while others advise a universal use of IVF/ICSI regardless of sperm characteristics. ${ }^{14}$

The objectives of this study were: (i) to assess sperm quality in cancer patients before being exposed to cytotoxic treatments; (ii) to evaluate the effect of different types of malignant diseases on sperm quality and follicle-stimulating hormone (FSH) levels; (iii) to investigate the rate of use of cryostored semen of cancer patients in a single large sperm bank in Israel; and (iv) to review the outcomes of ARTs in relation to the type of treatment and the type of malignancy.

\section{MATERIALS AND METHODS}

Patients and treatments

A total of 682 male cancer patients were referred for sperm cryopreservation in our sperm bank during a period of 20 years due to scheduled treatments with gonadotoxic potential. Testicular cancer (seminoma and non-seminoma tumors) had been diagnosed in 216 patients, lymphoma (either Hodgkin's or non-Hodgkin's) in 241 and various other types of cancer in 225 . All semen samples underwent cryopreservation, except for those from azoospermic men but 
regardless of the oncological treatment status. Seventy men returned throughout the years to withdraw their semen for fertility treatments. Two patients that made use of fresh sperm in combination with frozen-thawed sperm and four men for whom information was incomplete were excluded, as were two men who had non-cancerous disease (aplastic anemia). The remaining 62 men comprised the cohort of the current study.

Treatments included IUI cycles that were performed according to the quality of the thawed sperm (post thaw $>5$ million progressive motile sperm), IVF cycles (all performed before 1995 when ICSI was introduced in our center) and ICSI cycles (four of them with egg donation).

\section{Semen collection and freezing}

Ejaculates were obtained by masturbation, and a small sample was analyzed for relevant data that were recorded in the patient's file. Semen analysis procedure was described in detail in previous publication by our laboratory. ${ }^{15}$ We followed the guidelines of WHO guidelines ${ }^{16,17}$ of 1992 and 1999 that have not been critically changed during the study period. Each ejaculate intended for cryopreservation was diluted 1:1 with cryoprotectant (test yolk buffer with glycerol; Irvine Scientific, Santa Ana, CA, USA) and then divided into plastic straws that contained $0.5 \mathrm{ml}$ of fluid each (IMV, Paris, France). The eliquots were gradually cooled by vapor phase nitrogen suspension in a semiprogrammable freezer (Nicool LM-10; Air Liquid, Paris, France) at a rate of $1.7{ }^{\circ} \mathrm{C} \mathrm{min}^{-1}$, starting at room temperature and reaching $-7{ }^{\circ} \mathrm{C}$. The eliquots were further cooled at an increased rate of $-5{ }^{\circ} \mathrm{C}$ min $^{-1}$ until a temperature of $-100{ }^{\circ} \mathrm{C}$ had been reached, after which they were transferred into liquid nitrogen containers $\left(-196^{\circ} \mathrm{C}\right)$, as previously described. ${ }^{15} \mathrm{~A}$ post-thaw test fraction was then performed to evaluate the 'survival' rate of sperm under cryopreservation.

\section{Data collection}

Information for the current investigation was collected from the files in the sperm bank archives, the fertilization clinic's records and from the patients themselves. The retrieved data included the patient's age and oncological diagnosis at presentation, the type of oncological treatment that was scheduled/provided, parameters of sperm quality, the number and content of sperm samples that had been frozen, the ARTs that were used, and the details and outcome of the ART cycles. Pregnancy was defined whenever beta human chorionic gonadotropin was positive, including abortions, chemical and extruterine pregnancies. The retrospective study was approved by the institutional Helsinki committee for medical research involving human subjects.

\section{Statistical methods}

We measured mean values and standard deviation (s.d.) for various parameters, and examined the correlation of each variable with the ART results (pregnancy and delivery) by univariate comparison and logistic regression analysis using appropriate models for the group (according to $R$-square). Categorical variables of diagnostic groups, including different cancer types and ART modalities, were compared on using the chi-square and the Fisher's exact test (in cases where the group contained fewer than five events). The $t$-test and the non-parametric Mann-Whitney test were used to compare between two groups according to the achievement of pregnancy (or delivery). Categorical non-parametric rank tests and analysis of variance using post hoc tests adjusting for unequal variance and multiple comparisons were used for statistical analysis as appropriate. Differences were considered as significant when $P<0.05$. Analysis of data was performed using the Statistics Package for Social Sciences (SPSS for Windows version 11.0; SPSS, Chicago, IL, USA).

\section{RESULTS}

\section{Sperm parameters in cancer patients}

A total of 268 ejaculates had been frozen for the 70 men who returned to withdraw their sperm. The post-thaw test fraction was also performed on the same day of cryopreservation. Concentration was normal $\left(57.7 \pm 60.2\right.$ million $\left.\mathrm{ml}^{-1}\right)$, but motility was below the normal values both at prefreezing $(36.7 \% \pm 15.9 \%)$ and at post-thaw $(19.0 \% \pm 11.1 \%)$. The average total motile count was $62.6 \pm 76.9$ million, but the high value of s.d. signified the marked variability of the cryostored ejaculates. The post-thaw progressive motile concentration was also low $\left(4.7 \pm 7.6\right.$ million $\left.\mathrm{ml}^{-1}\right)$.

Morphology of the prefreezing samples was measured according to the WHO parameters ${ }^{18}$ until 1992, and then by the Kruger strict criteria. ${ }^{19}$ Sperm samples were divided into good (above $45 \%$ and above $14 \%$, respectively), medium $(21 \%-45 \%$ and $5 \%-13 \%$, respectively) and poor (below $20 \%$ and below $5 \%$, respectively). The distribution of morphology of the samples was: $20.6 \%$ good, $72 \%$ medium and $7.3 \%$ poor.

\section{Cancer types and sperm quality}

The sperm quality of the first ejaculates was compared between the different cancer types (Table 1). Testicular cancer patients were found to have the poorest sperm quality: their average sperm concentration was significantly lower than that of both the lymphoma patients and the 'other malignancies' patients $(P=0.04)$. The other variables of motility and post-thaw progressive motile concentration were not significantly different between the three groups.

The average baseline FSH levels were normal 7.3 $\pm 6.4 \mathrm{IU} \mathrm{l}^{-1}$ (normal range, $\left.1-9 \mathrm{IU}^{-1}\right)$. After completion of the cytotoxic treatments, the FSH levels reached as high as $21.4 \pm 6.6 \mathrm{IU}^{-1}$ (range 9.3-34 $\mathrm{IU} \mathrm{l}^{-1}$; $P<0.05$ ). Higher FSH levels were usually correlated with severe oligozoospermia or azoospermia. FSH levels continued to remain high, measured 12 years after completion of the treatment. FSH levels of patients with testicular cancer were above the normal range even before the cytotoxic treatment $\left(11.2 \pm 9.3 \mathrm{IU} 1^{-1}\right.$, compared to $6.2 \pm 4.4 \mathrm{IU} \mathrm{l}^{-1}$ for lymphoma patients and $5.3 \pm 2.8 \mathrm{IU}^{-1}$ for the other malignancies $(P=0.04$, analysis of variance $)$ ).

Table 1 Comparison of sperm quality among the groups of different cancer types

\begin{tabular}{|c|c|c|c|c|}
\hline \multirow{2}{*}{ Variable } & \multicolumn{3}{|c|}{ Malignancy type } & \multirow[b]{2}{*}{$P^{b}$} \\
\hline & Testicular $(\mathrm{n}=17)$ & Lymphoma $(\mathrm{n}=22)$ & Others $(n=23)$ & \\
\hline Sperm concentration $\left(\times 10^{6} \mathrm{ml}^{-1}\right)$ & $38.4 \pm 38.3$ & $69.4 \pm 51.1$ & $80.7 \pm 58.8$ & 0.04 \\
\hline Motility (\%) & $35.7 \pm 19.9$ & $42.5 \pm 11.7$ & $39.4 \pm 17$ & 0.6 \\
\hline Post-thaw progressive motile concentration $\left(\times 10^{6} \mathrm{ml}^{-1}\right)$ & $2.7 \pm 4.7$ & $6.6 \pm 2.8$ & $3.3 \pm 3$ & 0.2 \\
\hline
\end{tabular}

a Values given as mean \pm s.d.

${ }^{\mathrm{b}}$ Analysis of variance comparing the three groups. 


\section{The rate of cryo-thawed sperm usage for fertility treatments} Over the past 20 years, 70 patients out of 682 whose ejaculates were cryopreserved returned to withdraw their sperm in order to achieve pregnancy, yielding a rate of $10.3 \%$ for our unit. Eight men were excluded, and the age of the remaining 62 at the time of presentation ranged between 18 and 57 years (average 31.0 years). They had been diagnosed as having testicular cancer $(n=17)$, Hodgkin's and nonHodgkin's lymphoma $(n=22)$ and other kinds of malignant diseases $(n=23)$.

Most of the subjects $(51 / 62,82 \%)$ first applied to thaw their sperm within 4 years from the time of freezing, with the largest number of them $(19 / 62,31 \%)$ doing so during the first year. There was a gradual decrease in the number of first-time use of sperm over time: starting from the sixth year, only one patient returned each year for the firsttime use of his sperm (with an exception of two patients during the tenth year). The longest period of time sperm remained before being in this group was 13 years, and conception was achieved with that thawed semen during the thirteenth year (Figure 1).

\section{ART cycles and their outcomes}

A total of 207 ART cycles were reviewed, of which 154 (74.4\%) were carried out at the fertility unit in our medical center and 53 in other reproductive centers. Five cycles were excluded for lack of availability of relevant information, as some patients were lost to follow-up. Of the 184 cycles for which the results were fully available, conceptions were achieved in 46 (25.0\%), with 36 (19.6\%) of the pregnancies resulting in delivery. Three pregnancies were chemical, four ended as spontaneous abortions and three were extrauterine.

\section{Outcomes by ART type}

The study group of 62 patients and their partners underwent a total of 90 IUI cycles, 17 IVF cycles and 95 ICSI cycles (four of the latter with egg donation). Frozen-thawed cycles were merged with the original egg retrieval cycle in order to minimize a potential bias of the results. The ART data are summarized in Table 2. Post-thaw progressive motile sperm concentration in the IUI group was 2.5-20 million $\mathrm{ml}^{-1}$ (average 8.4 \pm 2.9 million $\mathrm{ml}^{-1}$ ).

Of the 24 couples treated with IUI information was available for 22 only. Of the nine pregnancies achieved by IUI, seven ended in deliveries, one was still ongoing and one was a chemical pregnancy $(8.6 \%$ deliveries per couple, Table 2). One couple had two deliveries $(31.8 \%$ $(8 / 22)$ pregnancy per couple rate, and $27.3 \%(6 / 22)$ deliveries per couple, Table 2 ) and one couple had a twin delivery. The minimal post-thaw progressive motile sperm concentration that resulted in a pregnancy was 6.7 million $\mathrm{ml}^{-1}$. Ovulation inductions with

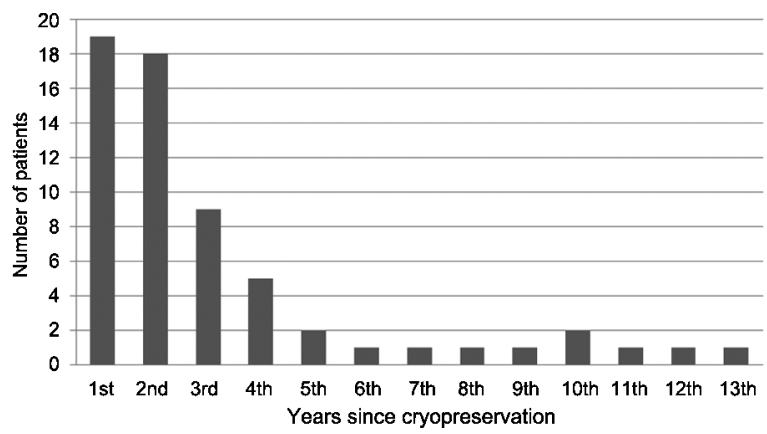

Figure 1 First withdrawal of sperm from the sperm bank according to years of cryopreservation. clomiphene citrate (three pregnancies) or gonadotropins (five pregnancies) were used in all but one of these cases.

Our unit used IVF from 1988 to 1995. Post-thaw progressive motile sperm concentration averaged 6.1 55.0 million $\mathrm{ml}^{-1}\left(0.4-17\right.$ million $\left.\mathrm{ml}^{-1}\right)$. None of those cycles resulted in pregnancy. The lack of information on two of the 10 couples (undergoing five cycles) might have biased our findings. ICSI technology was introduced in 1995: overall 1091 oocytes were aspirated of which 876 were injected (an average of 9.6 per cycle). Of the 41 couples treated with ICSI information was available for 38 only. Thirty-four pregnancies were achieved (37.4\% (34/ $91)$ per cycle) in 26 couples who had at least one pregnancy $(68.4 \%$, $26 / 38)$ and resulted in 23 deliveries (25.3\% (23/91) per cycle and $55.3 \%$ (21/38) per couple as two couples had two deliveries), including seven sets of twins. Two pregnancies were still ongoing, four ended with abortions, two were chemical pregnancies and three were extrauterine pregnancies (Table 2). There was no significant difference in the outcome of ART cycles of the different cancer groups (Table 3 ).

\section{DISCUSSION}

The current study summarizes more than 20 years of experience in sperm cryopreservation for cancer patients in a single tertiary center in Israel. It specifically addresses the issue of sperm usage and the outcome of various treatments. Only few publications are available in the literature on the fertilization results from the thawed sperm of former cancer patients because of the low percentage of men who have thus far returned to claim their cryopreserved sperm for the purpose of impregnation.

The $10.3 \%$ rate of thawed sperm usage in our center is among the highest reported, which was usually between $3 \%$ and $10 \% .^{20-25}$ The process of sperm cryopreservation for cancer patients mandates a complex coordinated procedure which is expensive, time consuming and emotional for the patients and demanding on the part of health providers. Current technology allows the freezing of sperm samples for decades, and this capability raises a number of questions concerning how long sperm should be preserved. Will there still be a demand for the sperm after many years? What is the actual yield of thawed sperm in terms of pregnancy achievement after many years? The data presented in this study, although retrospective, may assist in answering these questions. Based on these data, the rate of usage was the highest within the first year of cryopreservation $(30.6 \%)$, and $82.3 \%$ of the patients first returned to claim their sperm within 4 years. Use of frozen sperm gradually decreased with time during the first 5 years, remaining steady and in very small numbers thereafter (Figure 1). Similar findings were shown by Kelleher et al. ${ }^{26}: 70 \%$ of their 64 patients used their frozen-thawed sperm for fertility treatments within 5 years, $89 \%$ within 10 years and almost all (98\%) within 12 years. This pattern could probably be explained by the fact that during the period of time immediately after starting anticancer treatments, most patients experience a sharp decrease in their sperm counts and some even present with total azoospermia, making cryothawed sperm their only option to achieve a pregnancy. Moreover, these patients are usually advised by their physicians to actively avoid conceiving by ejaculated sperm for at least 1 year from the exposure of their sperm to cytotoxic treatments.

Some men may regain normal sperm production and be able to achieve spontaneous pregnancies, while those with irreversible posttreatment azoospermia will always be entirely dependent on their frozen-thawed sperm. Importantly, some couples return to claim their cryopreseved sperm in order to achieve additional pregnancies for many years after the samples have been deposited. For example, one 
Table 2 Outcome of treatment by different ART using thawed sperm of cancer patients

\begin{tabular}{|c|c|c|c|c|c|c|c|c|c|}
\hline $\begin{array}{l}\text { ART } \\
\text { type }\end{array}$ & $\begin{array}{c}\text { No. of couples with } \\
\text { known outcomes } \\
\text { (all couples) }\end{array}$ & $\begin{array}{l}\text { Cycles with } \\
\text { known outcomes } \\
\text { (all cycles) }\end{array}$ & $\begin{array}{c}\text { No. of } \\
\text { pregnancies }\end{array}$ & $F R^{\mathrm{a}}(\%)$ & $I R^{\mathrm{a}}(\%)$ & $\begin{array}{c}\text { Pregnancy per } \\
\text { cycle }^{\mathrm{b}}(\%)\end{array}$ & $\begin{array}{l}\text { Pregnancy per } \\
\text { couple }^{\mathrm{b}}(\%)\end{array}$ & $\begin{array}{c}\text { Deliveries per } \\
\text { cycle }^{c}(\%)\end{array}$ & $\begin{array}{c}\text { Deliveries per } \\
\text { couple }^{c}(\%)\end{array}$ \\
\hline IUI & $22(24)$ & $81(90)$ & 9 & & & 11.1 & 31.8 & 8.6 & 27.3 \\
\hline ICSI & $38(41)$ & $91(95)$ & 34 & 62.1 & 13.8 & 37.4 & 68.4 & 25.3 & 55.3 \\
\hline
\end{tabular}

Abbreviations: ART, assisted reproduction treatment; FR, fertilization rate; ICSI, intracytoplasmic sperm injection; IR, implantation rate; IUI, intrauterine insemination; IVF, in vitro fertilization.

${ }^{a}$ Overall pregnancy and implantation rates are indicated, calculated for the total number of eggs injected (ICSI) or prepared for fertilization (IVF) and the embryos transferred, respectively.

${ }^{\mathrm{b}} P<0.001$ (Fisher's exact test).

${ }^{c} P=0.003$ (Fisher's exact test).

of our patients claimed his sperm for the first time more than 12 years after completing oncological treatments and eventually fathered two children by means of his cryothawed sperm. Case reports in the literature describe the achievement of pregnancies even after longer periods of cryopreservation. Feldschuh et al. ${ }^{27}$ reported a live birth using sperm that was cryopreserved for 28 years.

The average sperm parameters for volume and concentration that were measured before the initiation of oncological treatment were within the normal range for all of our study patients (only motility was lower than the normal value 37\%). Sperm quality, however, was highly variable, ranging from excellent to severe oligo-asthenozoospermia. This variability probably represents the recognized effect of the type of cancer, disease stage and even grade might have on sperm production. ${ }^{28-30}$ Morphology was affected to a greater degree than any other examined sperm variable: it was below normal in $72 \%$ of the patients and in the poorer range $(<20 \%$ according to WHO criteria or $5 \%$ by Kruger criteria) in $7 \%$. Reactive oxidative species were suggested as a posible cause of DNA fragmentation. ${ }^{31}$ In a previous report, we included a group of the patients with cancer included in the current study, and described a significantly higher rate of DNA fragmentation of sperm cells after thawing. Yet, by comparing these results to patients without cancer, it was sugested that sperm quality, and mainly cryofreezability is appearently the main cause rather than cancer itself. ${ }^{32}$ It is beyond the scope of our study to address this important issue in detail. Most of the publications on the sperm quality of cancer patients focus solely on volume, concentration and motility, and so information on the extent of the influence of an existing malignancy on sperm morphology is very sparse. It could be of interest to investigate these changes in semen quality and FSH levels in future studies. As was previously reported, the most pronounced effect of decrease in sperm quality was found among testicular cancer patients compared to men with other types of cancer. ${ }^{29}$ Although indices of sperm quality were almost within the normal range, men with testicular cancer had the highest amount of abnormal sperm samples, as demonstrated by a very wide range in s.d. Their average FSH levels were higher even before oncological treatment, a finding that probably signifies a more pronounced effect of this type of cancer on spermatogenesis. We found a sharp increase in FSH levels even after the completion of treatments (i.e., from 7.3 to $21.4 \mathrm{mIu} \mathrm{ml}^{-1}$ ). FSH levels, however, had not been found to be a good predictor for sperm quality and recovery. $^{33}$

We employed various ART methods to achieve pregnancy with our patients' cryo-thawed sperm. Pregnancy and delivery rates by ICSI and IUI were similar to other groups of 'healthy' infertile couples using these modalities. In the IUI group, the pregnancy rate per cycle and per couple was $11 \%$ and $32 \%$ while $37 \%$ and $68 \%$ in the ICSI group. In contrast, the IVF results were highly disappointing, with the low fertilization rate of $20 \%$ and no pregnancy. There are several possible reasons for these poor IVF results. First, IVF was used only before 1995: some of the cycles were done even before 1990, when results by IVF were lower compared to today. Secondly, we were unable to retrieve information on five of the 17 IVF cycles that were carried out years ago, a factor which could bias the analysis of our IVF results. Finally, since ICSI was not available in our center prior to 1995, some IVF treatments used sperm cells of a quality below current minimal requirements (IVF was the only treatment option available at that time). The same poorer results of IVF compared to ICSI or IUI were found by others. ${ }^{26,34}$ Some authors received better pregnancy rates with IVF than with IUI; ${ }^{24,35}$ however, more recent studies suggested eliminating IVF altogether and performing ICSI as their preferred method of treatment. ${ }^{22-25,36}$ Table 4 summarizes the available literature on the experience of others with various ART modalities.

We recommend beginning with either the simpler treatment mode such as IUI (only if there are enough stored sperm samples and adequate quality), or start with the most efficient treatment mode of ICSI as the first line of treatment.

This retrospective study describes 20 years of experience in sperm preservation of cancer patients. Some of these couples are currently cryopreserving up to a few dozen samples. Since this may impose an economic burden, there should be a balance between what is really

Table 3 Outcome of assisted reproduction technique cycles of the different cancer groups

\begin{tabular}{|c|c|c|c|c|c|c|}
\hline \multirow[b]{2}{*}{ Cancer group } & \multicolumn{3}{|c|}{ Intrauterine insemination } & \multicolumn{3}{|c|}{$|C S|$} \\
\hline & Couples (cycles) & $\begin{array}{c}\text { Post-thaw motile } \\
\text { concentration (mean } \pm \text { s.d.) }\end{array}$ & $\begin{array}{c}\text { Clinical pregnancies } \\
\text { (deliveries) }\end{array}$ & couples (cycles) & Fertilization rate & $\begin{array}{c}\text { Clinical pregnancy } \\
\text { (deliveries) }\end{array}$ \\
\hline Testicular cancer & $5(23)$ & $8.2 \pm 4.0$ & $1(1)$ & $13(31)$ & 63.7 & $15(12)$ \\
\hline Lymphoma & $11(40)$ & $12.6 \pm 11.5$ & $6(6)$ & $15(32)$ & 58.6 & $10(10)$ \\
\hline Other cancer & $8(18)$ & $14.3 \pm 17.7$ & $1(1)$ & $13(27)$ & 65.2 & $8(5)$ \\
\hline
\end{tabular}

Abbreviations: ICSI, intracytoplasmic sperm injection; s.d., standard deviation. 
Table 4 Studies of pregnancy rate by different assisted reproduction techniques

\begin{tabular}{|c|c|c|c|c|c|}
\hline & No. of cycles & No. of couples & Pregnancy rate per cycle (\%) IUI & Pregnancy rate per cycle (\%) IVF & Pregnancy rate per cycle (\%) ICSI \\
\hline Ragni et al., $2003^{34}$ & 88 & 28 & 7.5 & 0 & 26 \\
\hline Kelleher et al., $2001^{26}$ & 85 & 60 & 35 & 21 & 54.5 \\
\hline Agarwal, $2004^{35}$ & 87 & 29 & 7 & 23 & 37 \\
\hline Revel et al., $2005^{23}$ & 62 & 21 & 5.9 & & 42 \\
\hline Mesequer et al., $2006^{36}$ & 35 & & 20 & & 50 \\
\hline van Casteren et al., $2008^{24}$ & 101 & 37 & 14.2 & 25 & 30.2 \\
\hline
\end{tabular}

Abbreviations: ICSI, intracytoplasmic sperm injection; IUI, intrauterine insemination; IVF, in vitro fertilization.

${ }^{\text {a }}$ Also reported to have $25 \%$ success rate with ICSI-frozen embryo transfer.

needed and keeping enough sperm to enable the couple to realize their desire to have a large family.

\section{AUTHOR CONTRIBUTIONS}

$\mathrm{AB}, \mathrm{GP}, \mathrm{LY}, \mathrm{RH}$ and HY were responsible for the concept and framework of the paper. SK, AB, OL and RH participated in collecting and evaluating the data. $\mathrm{AB}, \mathrm{SK}$ and $\mathrm{RH}$ wrote the paper. $\mathrm{AB}, \mathrm{RH}, \mathrm{SEK}$ and LY were largely responsible for the drafting and final editing. All authors read and approved the final manuscript.

\section{COMPETING FINANCIAL INTERESTS}

All authors declare that there are no competing financial interests.

1 Nalesnik JG, Sabanegh ES Jr, Eng TY, Buchholtz TA. Fertility in male after treatment for stage 1 and 2A seminoma. Am J Clin Oncol 2004; 27: 584-8.

2 Ragheb AM, Sabanegh ES Jr. Male fertility-implications of anticancer treatment and strategies to mitigate gonadotoxicity. Anticancer Agents Med Chem 2010; 10: 92-102.

3 Malas S, Levin V, Sur RJ. Fertility in patients treated with radiotherapy following orchiectomy for testicular seminoma. Clin Oncol 1994; 6: 377-80.

4 Foster RS, McNulty A, Rubin LR. Fertility considerations in nerve-sparing retroperitoneal lymph-node dissection. World J Urol 1994; 12: 136-8.

5 Hansen PV, Trykker H, Svennekjear IL, Hvolby J. Long-term recovery of spermatogenesis after radiotherapy in patients with testicular cancer. Radiother Oncol 1990; 18: 117-25.

6 Pryzant RM, Meistrich ML, Wilson G. Long term reduction in sperm count after chemotherapy with and without radiation therapy for non-Hodgkin's lymphomas. J Clin Oncol 1993; 11: 239-47.

7 Lampe $\mathrm{H}$, Horwich A, Norman A. Fertility after chemotherapy for testicular germ cell cancers. J Clin Oncol 1997; 15: 239-45.

8 Kenny LB, Laufer MR, Grant FD, Grier H, Diller L. High risk of infertility and long term gonadal damage in males treated with high dose cyclophosphamide for sarcoma during childhood. Cancer 2001; 91: 613-21.

9 Howell SJ, Shalet SM. Testicular function following chemotherapy. Hum Reprod Update 2001; 7: 363-9.

10 Giwercman A, Petersen PM. Cancer and male infertility. Baillieres Best Practice Res Clin Endo Metab 2000; 14: 453-71.

11 Tal R, Botchan A, Hauser R, Yogev L, Paz G et al. Follow-up of sperm concentration and motility in patients with lymphoma. Hum Reprod 2000; 15: 1985-8.

12 Sanger WG, Armitage JO, Schmidt MA. Feasibility of semen cryopreservation in patients with malignant disease. JAMA 1980; 244: 789-90.

13 Khalifa E, Oehninger S, Acosta AA. Successful fertilization and pregnancy outcome in in-vitro fertilization using cryopreserved/thawed spermatozoa from patients with malignant diseases. Hum Reprod 1992; 7: 105-8.

14 Hallak J, Kolletis PN, Sekhon VS. Sperm cryopreservation in patients with in patients with testicular cancer. Urology 1999; 54: 894-9.

15 Yogev L, Kleiman ES, Shabtai E, Botchan A, Gamzu R et al. Seasonal variations in preand post-thaw donor sperm quality. Hum Reprod 2004; 19: 880-5.
16 World Health Organization. Laboratory manual for the examination of human semen and semen cervical mucus interaction. 3rd edn. Cambridge: Cambridge University Press; 1992.

17 World Health Organization. Laboratory manual for the examination of human semen and semen cervical mucus interaction. 4th edn. Cambridge: Cambridge University Press; 1999.

18 World Health Organization. Laboratory manual for the examination of human semen and semen cervical mucus interaction. 2nd edn. Cambridge: Cambridge University Press; 1987.

19 Kruger TF, Menkveld R, Stander FS, Lombard GJ, van der Merwe JP et al. Sperm morphology as a prognostic factor in in vitro fertilization (IVF). Fertil Steril 1986; 46: 1118-23.

20 Sanger WG, OIson JH, Sherman JK. Semen cryobanking for men with cancer-criteria change. Fertil Steril 1992; 58: 1024-7.

21 Lass A, Akagbosu F, Abusheikha N, Hassouneh M, Blayney M et al. A program of semen cryopreservation for patients with malignant disease in a tertiary infertility center: lessons from 8 years' experience. Hum Reprod 1998; 13: 3256-61.

22 Schmidt KL, Larsen E, Bangsb $\mid$ II S, Meinertz H, Carlsen E et al. Assisted reproduction in male cancer survivors: fertility treatment and outcome in 67 couples. Hum Reprod 2004; 19: 2806-10.

23 Revel A, Haimov-Kochman R, Porat A, Lewin A, Somon A et al. In vitro fertilizationintracytoplasmic sperm injection success rates with cryopreserved sperm from patients with malignant disease. Fertil Steril 2005; 84: 118-22.

24 van Casteren NJ, van Santbrink EJ, van Inzen W, Romijn JC, Dohle GR. Use rate and assisted reproduction technologies outcome of cryopreserved semen from 629 cancer patients. Fertil Steril 2008; 90: 2245-50.

25 Crha I, Ventruba P, Zakova J, Paser M, Kubesova B et al. Survival and infertility treatment in male cancer patients after sperm banking. Fertil Steril 2009; 91 : 2344-8.

26 Kelleher S, Wishart SM, Liu PY, Turner L, di Pierro I et al. Long term outcomes of elective human sperm cryostorage. Hum Reprod 2001; 16: 2632-9.

27 Feldschuh J, Brassel J, Durso N, Levine A. Successful sperm storage for 28 years. Fertil Steril 2005; 84: 1017

28 Botchan A, Hauser R, Yogev L, Gamzu R, Paz G et al. Testicular cancer and spermatogenesis. Hum Reprod 1997; 12: 755-8.

29 Botchan A, Hauser R, Gamzu R, Yogev L, Lessing JB et al. Sperm quality in Hodgkin's disease versus non-Hodgkin's lymphoma. Hum Reprod 1997; 12: 73-6.

30 Hallak J, Mahran AM, Agarwal A. Characteristics of cryopreserved semen from men with lymphoma. J Assist Reprod Genet 2000; 17: 591-4.

31 Murtoni M, Maggi M, Spinelli S, Filimberti E, Forti G et al. Spontaneous DNA fragmentation in swim-up selected human spermatozoa during long term incubation. J Androl 2003; 24: 253-62.

32 Edelstein A, Yavetz H, Kleiman SE, Botchan A, Hauser R et al. Deoxyribonucleic aciddamaged sperm in cryopreserved-thawed specimens from cancer patients and healthy men. Fertil Steril 2008; 90: 205-8.

$33 \mathrm{Kader} \mathrm{HA}$, Rostom AH. Follicle stimulating hormone levels as a predictor of recovery of spermatogenesis following cancer therapy. Clin Oncol 1991; 3: 37-40.

34 Ragni G, Somigliana E, Restelli L, Salvi R, Arnoldi M et al. Sperm banking and rate of assisted reproduction treatments: insights from a 15-year cryopreservation program for male cancer patients. Cancer 2003; 97: 1624-9.

35 Agarwal A, Ranganathan P, Kattal N, Pasqualotto F, Hallak S et al. Fertility after cancer: a prospective review of assisted reproductive outcome with banked semen specimens. Fertil Steril 2004; 81: 342-8.

36 Mesequer M, Molina N, Garcia-Velasco JA, Remohi J, Pellicer A et al. Sperm cryopreservation in oncological patients: a 14 years follow up study. Fertil Steril 2006; 85: 640-5. 